\title{
Effect of Integrated Yoga Therapy on Arterial Stiffness: A Pilot Study on Young and Older Adults with Obesity
}

\author{
Pusuluri Venkata Giri Kumara ${ }^{a}$ Sudheer Deshpande ${ }^{b} \quad$ Aniruddha Joshi $^{\mathrm{e}}$ \\ Pooja More $^{a}$ Amit Singh ${ }^{c}$ H.R. Nagendrad \\ a Division of Yoga and Physical Sciences, S-VYASA Yoga University, ${ }^{b}$ VYASA, Eknath Bhavan, \\ 'Arogyadhama, and d S-VYASA Yoga University, Bengaluru, and eAtreya Innovations Pvt Ltd., \\ Pune, India
}

Keywords

Arterial stiffness $\cdot$ Stiffness index $\cdot$ Reflection index $\cdot$ Nadi Tarangini

\begin{abstract}
Background/Aims: The studies on the effect of integrated yoga therapy have shown significant results in improving the overall health. However, the effect of integrated yoga therapy on arterial stiffness is not yet established. The aim of the study was to investigate the effect of integrated yoga therapy on arterial stiffness measured at the radial artery. Methods: A total of 18 participants were included in the study. The participants were divided into three groups based on their age and body mass index. There were 5 participants in group 1 who were considered as nonobese, 7 younger adults with obesity were included in group 2, and 6 older adults with obesity were included in group 3. A 1-week integrated approach of yoga therapy (IAYT) was given as an intervention to the participants. The pulse data was acquired from participants using Nadi Tarangini at the beginning and end of the yoga program. The stiffness index (SI) and reflection index (RI) were computed from the pulse data. The data were analyzed using the paired-samples $t$ test. Results: There was a significant reduction in $\mathrm{SI}$ ( $p<$ 0.05 ) after IAYT in young participants with obesity. There was a significant reduction in BMI after IAYT in older adults, but the SI was only significantly low in young adults. There were no significant changes in RI after IAYT. Conclusion: The pilot study has shown that arterial stiffness measured at the radial artery using Nadi Tarangini has decreased significantly after 1 week of IAYT in young adults with obesity compared to older adults.
\end{abstract}




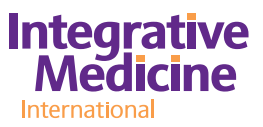

Integr Med Int 2017;4:85-93

DOI: $10.1159 / 000477542$

Venkata Giri Kumar et al.: Effect of Integrated Yoga Therapy on Arterial Stiffness: A Pilot Study on Young and Older Adults with Obesity

\section{Introduction}

As per the Taittiriya Upanishad, the human existence is a 5-layered body, namely Panchakosa consisting of Annamaya, Pranamaya, Manomaya, Vijnanamaya, and Anandamaya Kosas and the pathogenesis of psychosomatic diseases such as diabetes, hypertension, asthma, etc. can be well understood with the help of the Panchakosa model [1]. The integrated approach of yoga therapy (IAYT) developed by S-VYASA is a holistic approach to treat the diseases at all the layers of human existence and the studies based on IAYT have shown significant results in improving the overall status of health, which emphasizes the importance of holistic treatment rather than focused treatment [2-8]. The IAYT program includes therapies based on yoga, Ayurveda and naturopathy.

Arterial stiffness measured using the pulse wave velocity is considered as a potential indicator for cardiovascular risks and has gained significant clinical and research importance in the recent past [9]. The standard techniques used for measuring arterial stiffness are carotid-femoral pulse wave velocity, brachial-ankle pulse wave velocity, and photoplethysmography (PPG). Arterial stiffness measured using the carotid-femoral pulse wave velocity technique is considered as the gold standard [10] and as per this technique, it is measured as the ratio of the distance between two arteries (carotid and femoral) to the time taken for the pulse to travel from the carotid to the femoral artery. The other technique, i.e., brachial-ankle pulse wave velocity, is similar to carotid-femoral pulse wave velocity except for the pulse location and the clinical utility of arterial stiffness measured at the brachial artery has shown significant results in identifying cardiovascular risks [11]. PPG is a noninvasive technique which measures the changes in blood volume and the digital volume pulse (DVP) acquired using PPG has gained significant clinical importance in measuring oxygen saturation and blood pressure among others [12]. The DVP consists of systolic and diastolic peaks due to forward and reflected waves. The time interval between systolic and diastolic peaks depends on the stiffness of the arteries and height of the person. The ratio of the height of the person to the time interval between systolic and diastolic peaks is termed stiffness index (SI) which represents the arterial stiffness. The ratio of the diastolic peak amplitude to the systolic peak amplitude is termed reflection index (RI) which represents the endothelial function [13]. The effect of yoga on arterial stiffness has been studied by Patil et al. [14] and in their study they have reported that the yoga program reduced the arterial stiffness levels compared to brisk walk. Arterial stiffness has shown a close association with weight change such that weight gain leads to arterial stiffness progression and weight loss to arterial stiffness regression [15].

The effect of integrated yoga on changes in arterial stiffness is not yet established. As IAYT has shown significant results, we wanted to investigate the effect of a 1-week IAYT program on arterial stiffness measured from radial artery across young and older adults with obesity. The radial artery has been identified for measuring arterial stiffness as the pulse wave acquired from the radial artery is related to DVP acquired using PPG by a transfer function [16] and in another study arterial stiffness measured at the radial artery has shown a significant association with arterial stiffness measured using PPG [17]. We have identified Nadi Tarangini, a simple, convenient and noninvasive pulse acquisition system converting pulse pressure to electrical signal, for this study as it also measures pulse pressure using pressure sensors. The reproducibility, accuracy and precision of the instrument has been studied and the results were found to be consistent across multiple recordings at different times [18]. The studies on pulse rate variability [19], beat-to-beat alterations [20], and spectral analysis [21] using Nadi Tarangini have shown significant results. 
Table 1. Demographic details of the participants

\begin{tabular}{lccc}
\hline Parameter & Group 1 $(n=5)$ & Group 2 $(n=7)$ & Group 3 $(n=6)$ \\
\hline Age, years & $49.80 \pm 19.728$ & $26.57 \pm 11.745$ & $60.17 \pm 10.834$ \\
Height, cm & $161.20 \pm 3.347$ & $164.891 \pm 8.455$ & $157.823 \pm 3.136$ \\
Weight, kg & $58.236 \pm 10.054$ & $87.071 \pm 20.728$ & $80.203 \pm 14.934$ \\
BMI & $22.31 \pm 3.144$ & $32.159 \pm 7.859$ & $32.072 \pm 4.977$ \\
SBP, mm Hg & $123.20 \pm 11.454$ & $116.67 \pm 11.361$ & $133.33 \pm 15.002$ \\
DBP, mm Hg & $77.60 \pm 5.367$ & $76.67 \pm 13.486$ & $81.33 \pm 6.002$ \\
PR & $74.40 \pm 8.259$ & $81.33 \pm 12.044$ & $78.17 \pm 7.548$ \\
\hline
\end{tabular}

Data were represented as mean \pm standard deviation. Group 1, nonobese participants; group 2, younger adults with obesity; group 3, older participants with obesity. BMI, body mass index; SBP, systolic blood pressure; DBP, diastolic blood pressure; PR, pulse rate; $n$, number of participants.

\section{Materials and Methods}

\section{Participants}

A total of 18 participants ( 4 males and 14 females) from the Arogyadhama center (a residential center of S-VYASA) were included in the study. The participants have joined the Arogyadhama center to undergo a 1-week integrated yoga therapy. As the aim of the study was to investigate the effect of integrated yoga on arterial stiffness across young and old adults with obesity, participants were divided into three groups based on their age and body mass index (BMI). The participants with a BMI $<25$ were considered as normal, those with a BMI in the range of 25-30 were considered as overweight and those with a BMI $\geq 30$ were considered as obese. We included both overweight and obese participants in the obesity group. The participants with a BMI $<25$ were included in group 1, participants in the age range of 12-50 years with a BMI $\geq 25$ were included in group 2 , and participants above 50 years with a BMI $\geq 25$ were included in group 3 . The participants in group 1 were nonobese, the participants in group 2 were young adults with obesity, and the participants in group 3 were older adults with obesity. The participants in groups 1 and 3 had osteoarthritis as comorbidity. The medical history of the participants was examined by a residential doctor at the Arogyadhama center. The demographic details of the participants are provided in Table 1. Height, weight, blood pressure, and pulse rate were measured at the beginning and end of the yoga program. Blood pressure was measured using a standard mercury sphygmomanometer and pulse rate was measured manually by placing the fingers on the wrist. The BMI was computed as the ratio of weight to the square of height.

\section{Inclusion Criteria}

The participants who were not suffering from any severe cardiovascular diseases and who were not taking any medicines for cardiovascular diseases were included in the study.

\section{Exclusion Criteria}

We have excluded the participants who were suffering from cardiovascular diseases and who were not willing to be part of the study.

\section{Ethics Consideration}

The study was approved by the Institutional Ethics Committee. After having explained the aim of the study to the participants, we obtained informed consent from all them. 
Table 2. Integrated yoga program schedule

\begin{tabular}{|c|c|}
\hline Program & Description \\
\hline \multicolumn{2}{|c|}{ Integrated yoga program schedule for group 1 and group 3} \\
\hline Loosening exercises & toe, ankle, knee, waist, wrist, shoulder, neck rotation and bending sakti \\
\hline (10 rounds each) & $\begin{array}{l}\text { vikasaka sukshma vyayama for wrists, palms, fingers, elbows, arms, back, thighs } \\
\text { and calf muscles }\end{array}$ \\
\hline \multirow[t]{5}{*}{ Yogasanas (1-2 min) } & ardhakati cakrasana, ardha cakrasana, pada hastasana, bhujangasana, \\
\hline & salabhasana, dhanurasana, sarvangasana, matsyasana, viparitakarani, halasana, \\
\hline & cakrasana, sasankasana, vakrasana, ardha matsyendrasana, ustrasana, instant \\
\hline & relaxation technique ( $1 \mathrm{~min}$ ), quick relaxation technique (3 min), deep \\
\hline & relaxation technique (3 min) \\
\hline \multirow[t]{3}{*}{ Pranayama } & kapalbhati (40-120 strokes/min), sectional breathing (5 rounds), surya and \\
\hline & candra anuloma viloma pranayama (21 rounds), nadi sudhi pranayama (9 \\
\hline & rounds), cooling and bhramari pranayama (9 rounds) \\
\hline Meditation & nadanusandhana (3 min), OM meditation (15 min) \\
\hline Kriyas & jala neti, sutra neti, vamana dhouti \\
\hline \multicolumn{2}{|c|}{ Integrated yoga program schedule for group 2} \\
\hline \multirow{6}{*}{$\begin{array}{l}\text { Loosening exercises } \\
\text { (10 rounds each) }\end{array}$} & jogging, forward, backward and side bending, twisting, toe and heel touching, \\
\hline & knee rotation, pavanamuktasana kriya, rocking and rolling, alternate knee \\
\hline & touching, lumbar stretch, tiger stretch, back and side stretch, free walk, baby \\
\hline & walk, camel and crow walk, frog jump, step climbing, diagonal jumps, spinal \\
\hline & twist, jumping, surya namaskar (6-12 rounds), instant relaxation technique ( 2 \\
\hline & min), quick relaxation technique ( $2 \mathrm{~min}$ ), deep relaxation technique (6 min) \\
\hline Yogasanas & ardha padmasana, bhujangasana, salabhasana, dhanurasana, naukasana, \\
\hline (10 rounds) & navasana, parvatasana, vakrasana \\
\hline Pranayama & $\begin{array}{l}\text { sectional breathing ( } 5 \text { rounds), surya anuloma viloma pranayama ( } 27 \text { rounds), } \\
\text { nadi sudhi pranayama ( } 5 \text { rounds), bhramari pranayama ( } 5 \text { rounds) }\end{array}$ \\
\hline Meditation & nadanusandhana (9 rounds), OM meditation (15 min) \\
\hline Kriyas & kapalbhati, jal neti, sutra neti, vamana dhouti, sankha prakshalana \\
\hline
\end{tabular}

\section{Intervention}

A 1-week IAYT program was given as an intervention for the study. The program starts in the morning at 6 a.m. and ends at 7 p.m. The IAYT program includes kriyas, loosening exercises, asanas, pranayama, meditation, advanced yoga techniques, bhajans, and lectures on yoga philosophy. The yoga practices were rigorous for participants in group 2 when compared to the participants in groups 1 and 3 who had osteoarthritis as comorbidity. The details of yoga practices for all three groups are explained in Table 2. Kriyas were done once a week and the other yoga practices were done every day. There were two sessions of loosening exercises and asanas at 6 a.m. and 4 p.m. for a 1-h duration. Pranayama was done for $1 \mathrm{~h}$ at 10 a.m. Meditation and advanced yoga techniques were done for $1 \mathrm{~h}$ at $3 \mathrm{p} . \mathrm{m}$. and there were lectures on yoga philosophy for $1 \mathrm{~h}$ at 12 p.m. and bhajans for $1 \mathrm{~h}$ at 6 p.m. Apart from yoga practices, the IAYT program includes other therapies based on the concepts of Ayurveda and naturopathy consisting of valuka sveda, patrapinda sveda, shiro abhyangan, mustard pack, steam bath, sauna bath, mud bath, neutral underwater bath, hot hip bath, and circular jet bath. The participants were given 3 days of Ayurvedic therapies followed by 3 days of naturopathic therapies and the type of therapy was chosen by the doctor based on the medical condition of the participants. 
Fig. 1. Pulse wave acquired using Nadi Tarangini, representing various peaks and time periods of the pulse. P1, pulse amplitude at the systolic peak; P2, pulse amplitude at the inflection point; P3, pulse amplitude at the dicrotic notch; P4, pulse amplitude at the diastolic peak. Time periods T1, T2, T3, and $\mathrm{T} 4$ are measured from the start of the systolic phase. T1, time period at the systolic peak; $\mathrm{T} 2$, time period at the inflection point; T3, time period at the dicrotic notch; T4, time period at the diastolic peak.

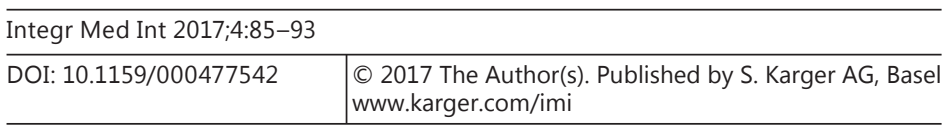

Venkata Giri Kumar et al.: Effect of Integrated Yoga Therapy on Arterial Stiffness: A Pilot Study on Young and Older Adults with Obesity

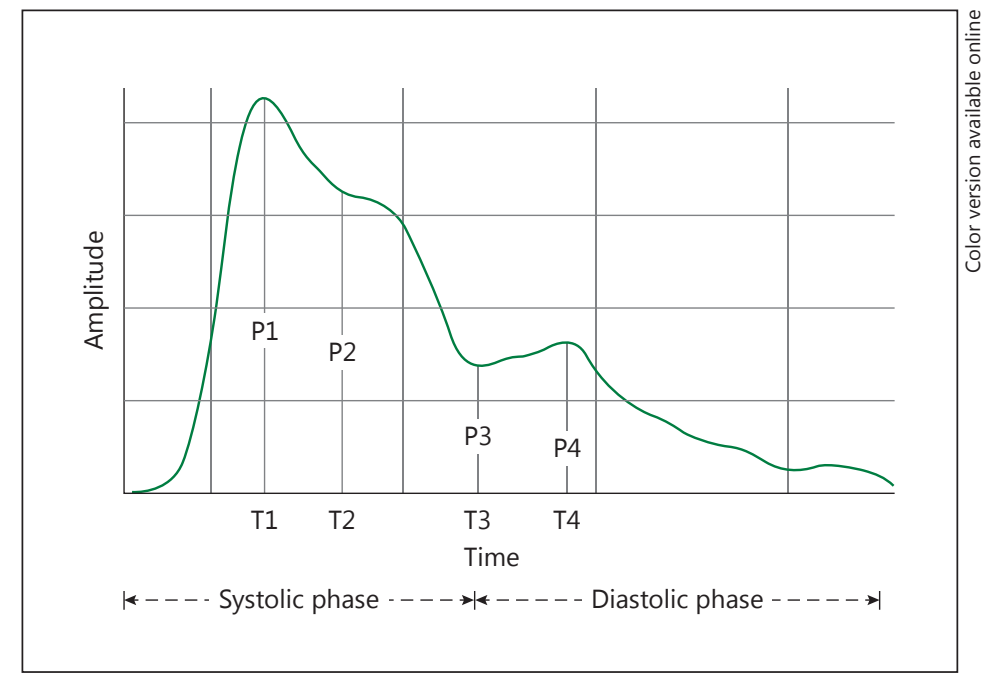

\section{Pulse Measurement}

Nadi Tarangini was used for collecting pulse data which has three linearly placed pressure transducers, a 16-bit multifunction data acquisition card NI USB-6210 (National Instruments, Austin, TX, USA) and LABVIEW, a data acquisition software. The pressure transducers of Nadi Tarangini convert pulse pressure to electrical signal, which is digitized by an analog-to-digital converter and the pulse data in the digital form will be stored on a personal computer. The pulse data acquired by Nadi Tarangini is a time series waveform representing pulse pressure. The pulse data were sampled at $425 \mathrm{~Hz}$. The three pressure sensors of the instrument measure the pulse at three dosha locations as defined by Ayurveda, namely vata, pitta and kapha. The pulse data were collected for $1 \mathrm{~min}$ by placing the sensors on the wrist. Initially, the pulse was sensed with fingers to identify the exact pulse locations and then the sensors were placed by closely aligning it with the sensed locations. The pulse was taken from the left hand for females and from the right hand for males. The pulse data consist of time and amplitudes of the pulse. As the pulse data get corrupted due to the noise induced by electrical and electronic sources, they were cleaned up using wavelet transformation. The pulse data were collected from the participants at the beginning of the IAYT and later at the end of the IAYT.

\section{Pulse Parameters}

The pulse data acquired by Nadi Tarangini were a continuous wave and Figure 1 corresponds to a single pulse wave isolated from the stream of pulse waves. We analyzed the stream of pulse waves by looking at the waveforms and for each individual we identified one pulse wave which has clear systolic and diastolic peaks. The pulse wave travels faster in stiff arteries compared to normal arteries and as a result the SI, which is a measure of arterial stiffness, increases with stiffness of arteries. The diastolic peak P4 at T4 corresponds to the reflected wave and systolic peak P1 at T1 corresponds to the forward wave.

The stiffness indices are computed as follows:

- $\quad \mathrm{SI}=$ height of the person $/(\mathrm{T} 4-\mathrm{T} 1)$

- $\quad \mathrm{RI}=$ diastolic peak / systolic peak (P4/P1).

\section{Statistical Analysis}

The data were analyzed using SPSS Statistics Version 10. They were presented as mean \pm standard deviation. The pulse data were assessed for normality using the Kolmogorov- 
Integrative
Medicine
International

Table 3. Summary of pairedsamples $t$ test

\begin{tabular}{l|l}
\hline Integr Med Int 2017;4:85-93 \\
\hline DOI: 10.1159/000477542 & $\begin{array}{l}\text { @ } 2017 \text { The Author(s). Published by S. Karger AG, Basel } \\
\text { www.karger.com/imi }\end{array}$ \\
\hline
\end{tabular}

Venkata Giri Kumar et al.: Effect of Integrated Yoga Therapy on Arterial Stiffness: A Pilot Study on Young and Older Adults with Obesity

\begin{tabular}{lccc}
\hline Group/parameter & Pre IAYT & Post IAYT & $p$ value \\
\hline Group 1 & & & \\
SI (m/s) & $17.488 \pm 6.2$ & $16.736 \pm 3.693$ & 0.779 \\
RI & $0.964 \pm 0.028$ & $0.970 \pm 0.029$ & 0.240 \\
BMI & $22.31 \pm 3.14$ & $21.995 \pm 2.903$ & 0.236 \\
SBP & $123.20 \pm 11.45$ & $112 \pm 11.225$ & $<0.01^{*}$ \\
DBP & $77.60 \pm 5.367$ & $75.60 \pm 6.066$ & 0.374 \\
PR & $74.40 \pm 8.295$ & $76.20 \pm 7.430$ & 0.748 \\
Group 2 & & & \\
SI (m/s) & $18.745 \pm 2.828$ & $16.072 \pm 1.731$ & $0.036^{*}$ \\
RI & $0.963 \pm 0.024$ & $0.966 \pm 0.025$ & 0.733 \\
BMI & $32.159 \pm 7.859$ & $31.154 \pm 7.029$ & 0.076 \\
SBP & $116.67 \pm 11.361$ & $114.83 \pm 5.307$ & 0.595 \\
DBP & $76.67 \pm 13.486$ & $75.67 \pm 8.335$ & 0.825 \\
PR & $81.33 \pm 12.044$ & $77.33 \pm 3.724$ & 0.488 \\
Group 3 & & & \\
SI (m/s) & $15.118 \pm 4.479$ & $13.606 \pm 4.404$ & 0.319 \\
RI & $0.941 \pm 0.043$ & $0.976 \pm 0.022$ & 0.057 \\
BMI & $32.072 \pm 4.977$ & $31.489 \pm 5.007$ & $0.019^{*}$ \\
SBP & $133.33 \pm 15.002$ & $125.67 \pm 5.854$ & 0.215 \\
DBP & $81.33 \pm 6.022$ & $75.33 \pm 6.022$ & $0.007^{*}$ \\
PR & $78.17 \pm 7.548$ & $78.50 \pm 0.837$ & 0.925 \\
\hline
\end{tabular}

Data were represented as mean \pm standard deviation. Group 1, nonobese participants; group 2, younger adults with obesity; group 3, older participants with obesity. SI, stiffness index; RI, reflection index; IAYT, integrated approach to yoga therapy; BMI, body mass index; SBP, systolic blood pressure; DBP, diastolic blood pressure; PR, pulse rate. * $p$ value comparing pre and post IAYT data, significance at 0.05 .

Smirnov test and the stiffness parameters SI and RI were found to be normal. The mean values of stiffness parameters (SI and RI) from pre- and post-IAYT were analyzed using the pairedsamples $t$ test in all three groups. The Pearson correlation coefficient was used to study the relationship between stiffness indices measured from pulse data and those measured from age and BMI. A two-tailed $p$ value $<0.05$ is considered statistically significant for all comparisons and the data were reported to three significant figures.

\section{Results and Discussion}

The mean values of SI and RI for pre- and post-IAYT are shown in Table 3. The obesity group had shown significant reduction in SI $(p<0.05)$ after IAYT, whereas there were no significant changes in the other groups. The RI was increased in all the groups but the change in the arthritis with obesity group was significant. In all the groups, the BMI was reduced after IAYT and the reduction in the arthritis with obesity group was significant. The correlations between stiffness indices (SI and RI) and age and BMI are summarized in Table 4. The SI and RI did not significantly correlate with age and BMI.

We studied the effect of IAYT on arterial stiffness measured at the radial artery using Nadi Tarangini. The pulse data with clear systolic and diastolic peaks were considered for the study. We analyzed the pulse data for proper systolic and diastolic peaks and observed that the pulse data at vata locations were having proper peaks for all the participants but the 


\section{Integrative Medicine \\ Internationa}

Table 4. Summary of correlations between SI, RI, age, BMI

\begin{tabular}{|c|c|c|c|c|}
\hline \multicolumn{5}{|c|}{ Integr Med Int 2017;4:85-93 } \\
\hline \multicolumn{2}{|c|}{ DOI: $10.1159 / 000477542$} & \multicolumn{3}{|c|}{$\begin{array}{l}\text { (c) } 2017 \text { The Author(s). Published by S. Karger AG, Base } \\
\text { www.karger.com/imi }\end{array}$} \\
\hline \multicolumn{5}{|c|}{$\begin{array}{l}\text { Venkata Giri Kumar et al.: Effect of Integrated Yoga Therapy on Arterial Stiffness: } \\
\text { A Pilot Study on Young and Older Adults with Obesity }\end{array}$} \\
\hline Parameter & Age & $p$ value* & BMI & $p$ value* \\
\hline \multicolumn{5}{|l|}{ Group 1} \\
\hline SI & 0.045 & 0.943 & 0.709 & 0.180 \\
\hline RI & -0.861 & 0.061 & -0.642 & 0.243 \\
\hline \multicolumn{5}{|l|}{ Group 2} \\
\hline SI & -0.114 & 0.808 & -0.487 & 0.268 \\
\hline RI & 0.128 & 0.785 & -0.197 & 0.672 \\
\hline \multicolumn{5}{|l|}{ Group 3} \\
\hline SI & -0.256 & 0.624 & 0.512 & 0.299 \\
\hline RI & -0.631 & 0.179 & 0.368 & 0.473 \\
\hline
\end{tabular}

SI, stiffness index; RI, reflection index; BMI, body mass index. Group 1 , nonobese participants; group 2, younger adults with obesity; group 3 , older participants with obesity. ${ }^{*} p$ value significance at 0.05 .

peaks were not proper at the pitta and kapha locations for many of the participants. This might be because the pulse had not been acquired properly at pitta and kapha locations as it would have been weak due to the deeply embedded artery at these locations. Secondly the sensors were equidistant and linearly placed and hence the sensors would not have been aligned precisely at the pulse locations on the wrist at these two locations. We considered pulse data at vata locations for our study; the pulse data corresponding to pitta and kapha were not included in the study as they were not having proper systolic and diastolic peaks.

In our pilot study, we observed that there was a significant reduction in arterial stiffness (SI) in young adults with obesity but the changes in older adults with obesity and nonobese adults were not significant after giving an intervention of IAYT for 1 week. Wildman et al. [22] in their study reported that the excess body weight is associated with aortic stiffness and in another study the authors have reported that weight change is associated with change in arterial stiffness [15]. In older adults and nonobese participants, there was a significant reduction in BMI but we did not see a significant reduction in SI and this could be because the average age of the group was above 50 years. The studies have shown that arterial stiffness increases with age [13] and hence for older adults with obesity the arterial stiffness increases with age as well as BMI, whereas in younger adults only obesity can be the cause of increase in arterial stiffness. In our study, results have shown that 1 week of IAYT program reduced the BMI of obese participants across young and older adults but a similar reduction in SI was observed only in young adults and not in older adults. We suggest that a 1-week duration may not have been sufficient for older adults to see significant changes in SI; secondly, older adults had osteoarthritis as a comorbidity and hence there is a need to study change in SI with IAYT by considering only obesity without any other complications. There was no significant change in RI after 1 week of IAYT in all the three groups, which needs further investigation.

In another study, Patil et al. [14] reported that the yoga program reduced the arterial stiffness levels when compared to brisk walk. In their study, arterial stiffness was assessed from pulse wave velocity measured using the carotid-femoral and brachial ankle technique, whereas in our study we assessed arterial stiffness at the radial artery. To our knowledge, this is the first time, the effect of integrated yoga therapy on arterial stiffness measured at the radial artery has been assessed. We have identified IAYT as an intervention for our study as previous studies with IAYT had shown significant improvements in the overall health of the patients $[7,8]$. The pilot study reported initial evidence of reduced arterial stiffness along with reduced BMI in young adults with obesity after 1 week of IAYT intervention. This needs to be further investigated with a larger sample size and with a control group in place. 


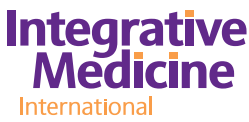

ntegrative
Medicine
Integr Med Int 2017;4:85-93

DOI: $10.1159 / 000477542$

2017 The Author(s). Published by S. Karger AG, Base www.karger.com/imi

Venkata Giri Kumar et al.: Effect of Integrated Yoga Therapy on Arterial Stiffness: A Pilot Study on Young and Older Adults with Obesity

In the present study, arterial stiffness was measured at the radial artery and we observed that 1 week of IAYT intervention reduced arterial stiffness in young adults with obesity compared to older adults and participants with normal weight. The pulse acquisition from the radial artery is simple and convenient compared to other techniques based on the carotidfemoral and brachial artery. Moreover, the pulse acquisition from the radial artery becomes much simpler and more sophisticated in the future as the semiconductor and sensor technologies are advancing further and coming up with precise sensors which can be mounted on the wrist with much more ease. Hence, there is a need to do in-depth studies in the future and establish the arterial stiffness measurements from the radial artery.

There were some limitations in our study. The focus of the pilot study was to investigate the effect of IAYT on arterial stiffness measured at the radial artery. As there were no prior studies in the literature, we have selected a relatively small sample size for our study. As the results were promising, we think that extensive studies with larger sample sizes would help in establishing the effect of integrated yoga on arterial stiffness. The second limitation was that IAYT was conducted for only 1 week and there is a need to study the changes in arterial stiffness by increasing the duration of IAYT. Another limitation of our study was that the yoga practices, diet, Ayurveda, and naturopathy treatments were not the same across the groups. As the pilot study reported a significant effect of IAYT on arterial stiffness, especially in younger adults, the future investigations should focus on studying the effect by giving the same yoga practices, diet, and treatments across the groups. The association of arterial stiffness with diabetes is well established [23] and hence there is a need to study the effect of yoga in general and IAYT in specific in mitigating the diabetic complications and improving the overall health of the patients with diabetes. However, in the current study, no control group was included and in the future, studies should include a control group to strengthen the study.

In conclusion, a 1-week IAYT intervention has significantly reduced arterial stiffness in young adults with obesity when compared to older adults. Arterial stiffness is considered as one of the potential cardiovascular risk factors and with a significant reduction in arterial stiffness we can say that a 1-week IAYT program can be suggested as an effective program to control the cardiovascular risk. In older adults, arterial stiffness also increases with age and hence it may take longer to see a similar effect. Therefore, the effect of IAYT on arterial stiffness needs to be studied with an extended duration of yoga therapy for older adults.

\section{Acknowledgements}

We express our sincere thanks to S-VYASA for supporting this study and all the volunteers of S-VYASA who helped us at various stages of the project. We sincerely thank Dr. Kashinath Metri and Dr. Judu Ilavarasu for reviewing the manuscript.

This research did not receive any specific grant from funding agencies in the public, commercial or not-for-profit sectors.

\section{Disclosure Statement}

The authors have no conflict of interest relevant to this article to report. 
Integrative Medicine

Venkata Giri Kumar et al.: Effect of Integrated Yoga Therapy on Arterial Stiffness: A Pilot Study on Young and Older Adults with Obesity

\section{References}

1 Nagarathna R, Nagendra HR: Yoga for Promotion of Positive Health. Bangalore, Swami Vivekananda Yoga Prakashana, 2000.

2 Villacres MC, Jagannathan A, Nagarathna R, Ramakrsihna J: Decoding the integrated approach to yoga therapy: qualitative evidence based conceptual framework. Int J Yoga 2014;7:22-31.

-3 Ebnezar J, Nagarathna R, Yogitha B, Nagendra H: Effect of integrated yoga therapy on pain, morning stiffness and anxiety in osteoarthritis of the knee joint: a randomized control study. Int J Yoga 2012;5:28-36.

$\checkmark 4$ Ebnezar J, YogithaBali M, John R, Gupta 0: Role of integrated approach of yoga therapy in a failed post-total knee replacement of bilateral knees. Int J Yoga 2014;7:160-164.

-5 Jagannathan A, Hamza A, Thirthalli J, Nagendra H, Nagarathna R, Gangadhar B: Development and feasibility of need-based yoga program for family caregivers of in-patients with schizophrenia in India. Int J Yoga 2012;5: 42-47.

-6 Radhakrishna S: Application of integrated yoga therapy to increase imitation skills in children with autism spectrum disorder. Int J Yoga 2010;3:26-30.

7 Ranjita R, Badhai S, Hankey A, Nagendra H: A randomized controlled study on assessment of health status, depression, and anxiety in coal miners with chronic obstructive pulmonary disease following yoga training. Int J Yoga 2016;9:137-144.

-8 Tekur P, Chametcha S, Hongasandra R, Raghuram N: Effect of yoga on quality of life of CLBP patients: a randomized control study. Int J Yoga 2010;3:10-17.

$\$ 9$ Avolio AP, Butlin M, Walsh A: Arterial blood pressure measurement and pulse wave analysis: their role in enhancing cardiovascular assessment. Physiol Meas 2010;31:R1-R47.

$\checkmark 10$ Mancia G, Fagard R, Narkiewicz K, Redon J, Zanchetti A, Böhm M, et al: 2013 ESH/ESC guidelines for the management of arterial hypertension: the Task Force for the management of arterial hypertension of the European Society of Hypertension (ESH) and of the European Society of Cardiology (ESC). Eur Heart J 2013; $34: 2159-2219$.

11 Sugawara J, Tanaka H: Brachial-ankle pulse wave velocity: myths, misconceptions, and realities. Pulse 2015; 3:106-113.

12 Hertzman AB: The blood supply of various skins areas as estimated by the photoelectric plethysmograph. Am J Physiol 1938;124:328-340.

13 Millasseau SC, Ritter JM, Takazawa K, Chowienczyk PJ: Contour analysis of the photoplethysmographic pulse measured at the finger. J Hypertens 2006;24:1449-1456.

14 Patil SG, Aithala MR, Das KK: Effect of yoga on arterial stiffness in elderly subjects with increased pulse pressure: a randomized controlled study. Complement Ther Med 2016;23:562-569.

15 Wildman RP, Farhat GN, Patel AS, Mackey RH, Brockwell S, Thompson T, et al: Weight change is associated with change in arterial stiffness among healthy young adults. Hypertension 2005;45:187-192.

16 Millasseau SC, Guigui FG, Kelly RP, Prasad K, Cockcroft JR, Ritter JM, et al: Noninvasive assessment of the digital volume pulse. Comparison with the peripheral pressure pulse. Hypertension 2000;36:952-956.

17 Wu HT, Lee CH, Liu AB, Chung WS, Tang CJ, Sun CK, et al: Arterial stiffness using radial arterial waveforms measured at the wrist as an indicator of diabetic control in the elderly. IEEE Trans Biomed Eng 2011;58:243252.

18 Joshi A, Kulkarni A, Chandran S, Jayaraman VK, Kulkarni BD: Nadi Tarangini: a pulse based diagnostic system. Conf Proc IEEE Eng Med Biol Soc 2007;2007:2207-2210.

19 Joshi AJ, Chandran S, Jayaraman VK, Kulkarni BD: Arterial pulse rate variability analysis for diagnoses. 19th Int Conf Pattern Recognit, Tampa, 2008.

20 Joshi A, Chandran S, Jayaraman VK, Kulkarni BD: Arterial pulse system: modern methods for traditional Indian medicine. Conf Proc IEEE Eng Med Biol Soc 2007;2007:608-611.

21 Joshi AJ, Chandran S, Jayaraman VK, Kulkarni BD: Multifractality in arterial pulse. 19th Int Conf Pattern Recognit, Tampa, 2008.

22 Wildman RP, Mackey RH, Bostom A, Thompson T, Sutton-Tyrrell K: Measures of obesity are associated with vascular stiffness in young and older adults. Hypertension 2003;42:468-473.

23 de Oliveira Alvim R, Mourao-Junior CA, de Oliveira CM, de Faria Lima R, Horimoto ARVR, Hong VAC, et al: Glycemic control and arterial stiffness in a Brazilian rural population: Baependi Heart Study. Diabetol Metab Syndr 2015;7:86. 